\title{
Development and Optimization of a Novel Sustained-release Tablet Formulation for Bupropion Hydrochloride using Box-Behnken Design
}

\author{
Kwang-Ho Cha, Nayoung Lee, Min-Soo Kim, Jeong-Soo Kim, Hee Jun Park, Junsung Park, \\ Wonkyung Cho and Sung-Joo Hwang \\ Center for Nanotechnology-based New Drug Dosage Form, College of Pharmacy, \\ Chungnam National University, 220 Gung-dong, Yuseong-gu, Daejeon, 305-764, Korea \\ (Received September 3, $2010 \cdot$ Revised October 8, $2010 \cdot$ Accepted October 9, 2010)
}

\begin{abstract}
The objectives of this study were to evaluate the effect of formulation ingredients on the drug release and to optimize the novel sustained release matrix tablet formulations of bupropion hydrochloride. A three factor, three-level Box-Behnken design was used for the optimization procedure, with the amounts of PEO $\left(X_{1}\right)$, citric acid $\left(X_{2}\right)$ and Compritol 888 ATO $\left(X_{3}\right)$ as the independent variables. The selected dependent variables were the cumulative percentage values of bupropion hydrochloride that had dissolved after 1, 4 and $8 \mathrm{hr}$. Various dissolution profiles of the drug from sustained release matrix tablets were obtained. Optimization was performed for $X_{1}, X_{2}$ and $X_{3}$ using the following target ranges; $30 \% \leq Y_{1} \leq 45 \% ; 70 \leq Y_{2} \leq 85 \% ; 85 \% \leq Y_{3} \leq 100 \%$. The optimized formulation for bupropion hydrochloride was achieved with $12.5 \%$ PEO $\left(X_{1}\right), 2.5 \%$ citric acid $\left(X_{2}\right)$ and $10 \%$ Compritol 888 ATO $\left(X_{3}\right)$. The sustained release matrix tablets with the optimized formulation provided a release profile that was close to predicted values. In addition, the dissolution profiles of the sustained release matrix tablet with the optimized formulation were similar to those of the commercial product Wellbutrin ${ }^{\mathbb{R}}$ SR tablets $\left(f_{2}=79.83\right)$.
\end{abstract}

Key words - Bupropion, Sustained-release tablet, Box-Behnken design

Bupropion hydrochloride (BPH) is an aminoketone-derivative chemically unrelated to other currently available antidepressants (e.g., selective serotonin reuptake inhibitors, tricyclics and tetracyclics). It inhibits the neuronal reuptake of norepinephrine and dopamine and does not inhibit monoamine oxidase (Ascher et al., 1995; Ferris and Cooper, 1993). BPH is the purported active ingredient in the antidepressant and the smoking cessation. Moreover, BPH is also being considered as a potential treatment for attention deficit hyperactivity disorder (Barrickman et al., 1995; Cyr and Brown, 1998) and obesity (Palamara et al., 2006). But, its neurochemical mechanisms of the antidepressant and smoking cessation effects are unknown.

Immediate-release BPH (Wellbutrin ${ }^{\circledR} 75 \mathrm{mg}, 100 \mathrm{mg}$ ) has been on the market since 1989 and is usually well-tolerated antidepressant. However, there were seizures in approximately $0.4 \%(4 / 1,000)$ of patients treated at doses up to $450 \mathrm{mg} /$ day. In order to reduce the adverse effect, sustained-release BPH (Wellbutrin ${ }^{\circledR}$ SR 100mg, 150mg, 200mg) has been developed in 1996 (Settle, 1998). The Pharmacokinetic studies indicated that BPH considered a suitable candidate for sustained-release formulations (Jefferson et al., 2005). Wellbutrin ${ }^{\circledR}$ SR tablets

\footnotetext{
Corresponding Author :

Tel : +82-42-821-5922, E-mail : sjhwang@cnu.ac.kr

DOI : $10.4333 /$ KPS.2010.40.5.313
}

comprise a HPMC based matrix core containing cysteine hydrochloride as a stabilizer and film coating.

Polymeric materials have been widely used in order to modify and modulate the drug release from solid pharmaceutical dosage forms such as sustained-release or controlled-release matrix tablet (Ford et al., 1987). From the wide choice of possible matrix materials, PEO (Poly ethylene oxide), citric acid, Compritol 888 ATO (glyceryl behenate) has been used in the formulation of sustained release monolithic matrix tablets. PEO is the most widely used in controlled release matrix tablets, owing to its solubility in water, availability in a range of molecular weight/viscosity grades and unique swelling/erosion characteristics which can be utilized in modulating drug release profiles (Jamzad and Fassihi, 2006). And the addition of some organic acids to matrix tablets has been used to modify the release rate of drugs. The addition of citric acid contributes to maintain a low $\mathrm{pH}$ inside the matrix and acts loosening the matrix structure through an increased porosity created after its dissolution and release (González and Robles, 2003; Kranz et al., 2005). Lipid excipients are also classically used for the preparation of sustained-release formulations due to their lipophilic properties. In this study, Compritol 888 ATO among other lipid excipients used to prevent from interaction between drug and hydrophilic excipients using hot melt coating method. Because, BPH has highly hygroscopic nature and 
$\mathrm{BPH}$ is susceptible to degradation in high $\mathrm{pH}$.

Statistical experimental design methodologies are powerful, efficient and systematic tools in the design of pharmaceutical dosage forms, allowing a rational study of the influence of formulation and/or processing parameters on the selected responses with a shortening of the experiment time and an improvement in the research and development work (Furlanetto et al., 2006). The objective of this study was to evaluate the effect of formulation ingredients (amount of PEO, citric acid and Compritol 888 ATO) on the BPH drug release and to optimize the novel sustained release matrix tablet formulations of BPH based on response surface methodology (RSM) utilizing polynomial equation. The selected response variables were the cumulative $\%$ drug release in different time points (1, 4 and $8 \mathrm{hr}$ ).

\section{Materials and Method}

\section{Material}

The following materials were gifted: bupropion hydrochloride (Sinoway Industrial Co. Ltd. Xiamen, China), polyeth-

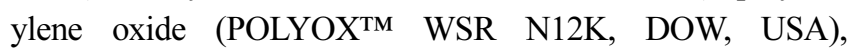
Compritol 888 ATO (glycryl behenate NF, Gattefossé, St. Priest, France), citric acid (Sigma, USA), microcrystalline cellulose (Avicel ${ }^{\circledR}$ PH102, FMC, USA) and magnesium stearate (Joinway Pharm, Zhejiang, China). Wellbutrin SR ${ }^{\circledR}$ tablets (Lot No. NM0026, GlaxoSmithKline Korea, Seoul, Korea) were purchased from the market. All organic solvents were of high performance liquid chromatography (HPLC) grade. All other chemicals were of reagent grade.

\section{Experimental design}

A three-factor, three-level Box-Behnken design was used for the optimization procedure with PEO content $\left(X_{1}\right)$, citric acid content $\left(X_{2}\right)$ and Compritol 888 ATO content $\left(X_{3}\right)$ as the independent variables. The levels for these three parameters were determined from the preliminary trials. The percentages of the drug released at 1, 4 and $8 \mathrm{hr}$ were used as dependent variables for desirable drug release. Table I summarizes the factors, the levels tested, and the responses. PEO content, citric acid content and Compritol 888 ATO content were determined in the range of $12.5-25 \%(\mathrm{w} / \mathrm{w}), 0-5 \%(\mathrm{w} / \mathrm{w})$ and $0-10 \%(\mathrm{w} / \mathrm{w})$, respectively. From the data obtained, response surfaces were constructed using the software package Design Expert software (version 7.0, Stat-Ease Inc., Minneapolis, U.S.A.). A suitable polynomial model was selected based on the estimation of several statistical parameters such as the multiple correlation coefficient $\left(R^{2}\right)$, adjusted multiple correlation coefficient (adjusted
Table I. Variables in Box-Behnken Design

\begin{tabular}{lccc}
\hline \hline \multirow{2}{*}{ Formulation variables } & \multicolumn{3}{c}{ Level used } \\
\cline { 2 - 4 } & -1 & 0 & 1 \\
\hline$X_{1}=$ PEO content (mg) & 50 & 75 & 100 \\
$X_{2}=$ Citric acid content (mg) & 0 & 10 & 20 \\
$X_{3}=$ Compritol 888 ATO content (mg) & 0 & 20 & 40 \\
Response variables & \multicolumn{3}{c}{ Constraints } \\
$Y_{1}=$ cumulative \% drug dissolved & $30 \% \leq Y_{1} \leq 45 \%$ \\
$\quad$ in $1 \mathrm{hr}$ & \multicolumn{2}{|c}{$70 \% \leq Y_{2} \leq 85 \%$} \\
$Y_{2}=$ & cumulative \% drug dissolved & in $4 \mathrm{hr}$ \\
$Y_{3}=$ & cumulative \% drug dissolved & \\
& in $8 \mathrm{hr}$
\end{tabular}

$R^{2}$ ) and the predicted residual sum of square (PRESS), also provides by Design-Expert software.

\section{Preparation of tablets}

Compritol 888 ATO was melted by water bath at $75^{\circ} \mathrm{C}$. BPH was added continuous stirring to get a homogeneous dispersion. Then, the molten mass was cooled to room temperature and resolidified. The resultant solid was pulverized and passed through a 30-mesh sieve (less than $500 \mu \mathrm{m}$ ).

Each formula contained a fix dose $(150 \mathrm{mg})$ of bupropion hydrochloride as the active ingredient. Proportions of hydrophilic PEO (12.5-25\%, w/w), citric acid (0-5\%, w/w) and lipid-based Compritol 888 ATO (0-10\%,w/w) were variable among the formulation, while magnesium stearate was maintained at a constant proportion $(3.75 \%$, w/w). The total tablet weight of the preparations was $400 \mathrm{mg}$, which was achieved with the additional use of tablet excipient microcrystalline cellulose. The components mixture was compressed into tablets using $11 \mathrm{~mm}$ punch by ERWEKA ${ }^{\circledR}$ EKO (Germany). The hardnesses for all formulations were adjusted to be in the range of 9-11 kP. The compositions of each matrix tablet formulation were prepared according to the experimental design (Table II).

\section{Dissolution studies}

The release of the drug from the matrix tablet was performed according to the USP paddle method (apparatus 2) using a dissolution apparatus (VanKel ${ }^{\circledR}$ VK 7000 Cary, NC). Dissolution study was conducted in $900 \mathrm{~mL}$ deionized water at $37 \pm 0.5^{\circ} \mathrm{C}$ and the rotation speed was set at $50 \mathrm{rpm}$. Dissolution progress was monitored at predetermined time intervals. After each interval, $2 \mathrm{~mL}$ dissolution medium was withdrawn and filtered immediately, and then $2 \mathrm{~mL}$ fresh medium was added in its place. BPH release was determined at wavelength of $298 \mathrm{~nm}$ by UV-spectrophotometer (Shimadzu mini-1240). 
Table II. Experimental Runs and Observed Responses for Box-Behnken Design

\begin{tabular}{ccccccc}
\hline \hline \multirow{2}{*}{ Run } & \multicolumn{3}{c}{ Factor } & \multicolumn{3}{c}{ Responses } \\
\cline { 2 - 7 } & $X_{1}$ & $X_{2}$ & $X_{3}$ & $Y_{1}$ & $Y_{2}$ & $Y_{3}$ \\
\hline 1 & 50 & 10 & 0 & 42.65 & 83.05 & 99.11 \\
2 & 100 & 10 & 40 & 27.44 & 66.27 & 92.73 \\
3 & 75 & 10 & 20 & 32.87 & 70.95 & 94.62 \\
4 & 50 & 10 & 40 & 38.18 & 80.14 & 99.03 \\
5 & 75 & 20 & 40 & 31.35 & 70.85 & 97.28 \\
6 & 50 & 20 & 20 & 39.85 & 80.6 & 98.81 \\
7 & 75 & 10 & 20 & 31.95 & 70.13 & 93.51 \\
8 & 75 & 0 & 40 & 31.81 & 64.56 & 75.9 \\
9 & 75 & 0 & 0 & 34.25 & 66.64 & 74.82 \\
10 & 75 & 20 & 0 & 37.25 & 79.74 & 99.12 \\
11 & 100 & 10 & 0 & 31.51 & 71.1 & 97.1 \\
12 & 75 & 10 & 20 & 32.56 & 69.42 & 98.58 \\
13 & 50 & 0 & 20 & 40.21 & 70.4 & 75.77 \\
14 & 100 & 0 & 20 & 28.86 & 60.25 & 77.22 \\
15 & 100 & 20 & 20 & 29.93 & 66.24 & 91.87 \\
\hline
\end{tabular}

The dissolution profiles of BPH were compared to those of Wellbutrin ${ }^{\circledR}$ SR containing similar amount of BPH using similarity factor $\left(f_{2}\right)$ described as following equation

$$
f_{2}=50 \times \log \left\{\left[1+\left(\frac{1}{n}\right) \sum_{t=1}^{n}\left|R_{t}-T_{t}\right|^{2}\right]^{-0.5} \times 100\right\}
$$

where $n$ is the number of time point, $R_{t}$ and $T_{t}$ are the percent dissolved of the reference and test products at each time point t. An $f_{2}$ value between 50 and 100 suggests that two dissolution profiles are similar (Moore and Flanner, 1996).

\section{Results and Discussion}

For the response surface methodology involving BoxBehnken design, a total of 15 experiments were performed for three factors at three levels each. This number is equal to the mid-point of each edge and the three replicated center points of the cube. The experiment runs with independent variables and the observed responses for the 15 formulations are shown in Fig. 1 and Table II. Based on the Box-Behnken model, the factor combinations resulted in different drug release rates. Various models, such as Linear, 2FI, Quadratic and Cubic, were fitted to the data for three responses simultaneously using Design Expert software and adequacy, and good fit of the model was tested using ANOVA. The $R^{2}$, adjusted $R^{2}$ and the PRESS, provided by Design-Expert software, were used as factors for selection of adequate models. As presented in Table
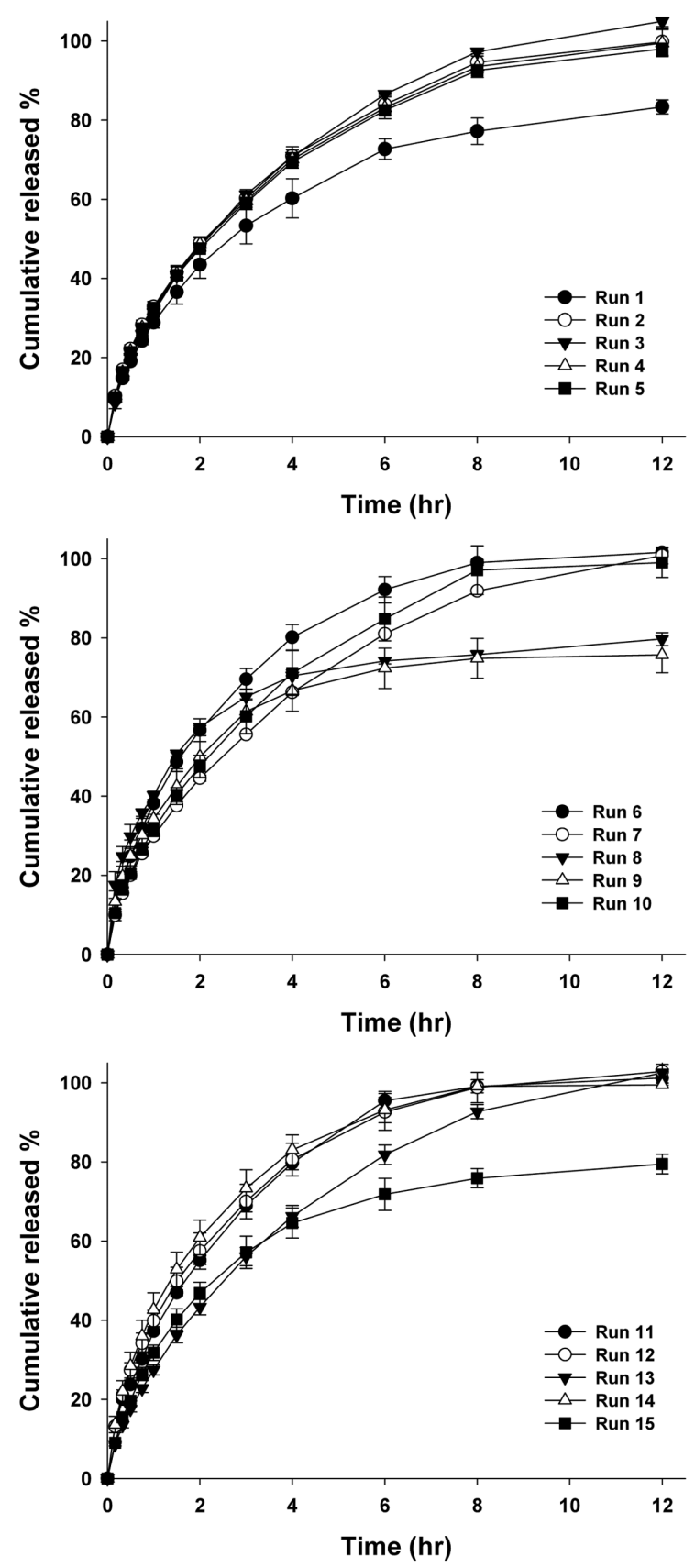

Figure 1. Dissolution profiles of all formulations in water $(n=6$, means \pm S.D.)

III, the quadratic model was selected as a suitable statistical model for optimized formulations because it had the smallest value of PRESS. PRESS is a measure of the fit of the model to the points in the design. The smaller the PRESS statistic is, the better the model fits to the data points (Segurola et al., 1999). The model showed a statistically insignificant lack of fit, as shown in Table III. The adequacy of the model was also confirmed with residual plot tests of regression models. ANOVA was applied to estimate the significance of the model 
Table III. Summary of Results of: (a) Model analysis, (b) Lack of fit and (c) R-square analysis for Measured Responses

\begin{tabular}{|c|c|c|c|c|c|c|}
\hline \multirow{2}{*}{ Source } & \multicolumn{2}{|c|}{$Y_{1}$} & \multicolumn{2}{|c|}{ 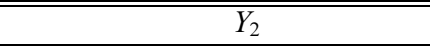 } & \multicolumn{2}{|c|}{ 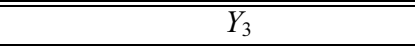 } \\
\hline & Sum of squares & $\mathrm{P}>\mathrm{F}$ & Sum of squares & $\mathrm{P}>\mathrm{F}$ & Sum of squares & $\mathrm{P}>\mathrm{F}$ \\
\hline \multicolumn{7}{|l|}{ (a) Model analysis } \\
\hline Mean vs. total & 17385.59 & & 76375.18 & & 124300 & \\
\hline Linear vs. mean & 269.98 & $<0.0001 * *$ & 518.64 & $0.0001 * *$ & 896.02 & $0.0049 * *$ \\
\hline $2 \mathrm{FI}^{\mathrm{a}}$ vs. linear & 3.54 & 0.5923 & 16.95 & 0.6714 & 24.33 & 0.9203 \\
\hline Quadratic vs. 2FI & 13.14 & $0.0021 * *$ & 80.56 & $0.0010 * *$ & 380.84 & $0.0019 * *$ \\
\hline Cubic vs. quadratic & 0.47 & 0.6276 & 2.80 & 0.4084 & 10.68 & 0.7190 \\
\hline Residual & 0.44 & & 1.17 & & 14.21 & \\
\hline Total & 17672.86 & & 76995.29 & & 125600 & \\
\hline \multicolumn{7}{|l|}{ (b) Lack of fit } \\
\hline Linear & 17.15 & 0.1073 & 100.30 & 0.0510 & 415.85 & 0.1403 \\
\hline $2 \mathrm{FI}$ & 13.61 & 0.0907 & 83.36 & $0.0410^{*}$ & 391.52 & 0.1014 \\
\hline Quadratic & 0.47 & 0.6276 & 2.80 & 0.4084 & 10.68 & 0.7190 \\
\hline Cubic & 0.000 & & 0.000 & & 0.000 & \\
\hline \multirow[t]{2}{*}{ Pure error } & 0.44 & & 1.17 & & 14.21 & \\
\hline & Adjusted $R$-square & PRESS & Adjusted $R$-square & PRESS & Adjusted $R$-square & PRESS \\
\hline \multicolumn{7}{|l|}{ (c) $R$-square analysis } \\
\hline Linear & 0.9221 & 29.75 & 0.7917 & 211.93 & 0.5872 & 845.48 \\
\hline $2 \mathrm{FI}$ & 0.9144 & 41.49 & 0.7615 & 427.52 & 0.4646 & 1843.15 \\
\hline Quadratic & 0.9911 & 8.51 & 0.9821 & 47.42 & 0.9475 & 202.78 \\
\hline Cubic & 0.9893 & $\mathrm{ND}^{\mathrm{b}}$ & 0.9868 & $\mathrm{ND}^{\mathrm{b}}$ & 0.9250 & $\mathrm{ND}^{\mathrm{b}}$ \\
\hline
\end{tabular}

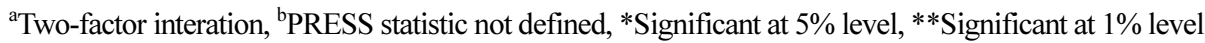

Table IV. Quadratic Equations for the Quantiative Effect of Independent Variables $\left(X_{1}, X_{2}, X_{3}\right)$ on Responses $\left(Y_{1}, Y_{2}, Y_{3}\right)$

\begin{tabular}{c}
$Y_{1}=32.46-5.39 X_{1}+0.41 X_{2}-2.11 X_{3}+0.36 X_{1} X_{2}+0.1 X_{1} X_{3}-0.86 X_{2} X_{3}+1.77 X_{1}^{2}+0.49 X_{2}^{2}+0.72 X_{3}^{2}$ \\
$Y_{2}=70.17-6.29 X_{1}+4.45 X_{2}-2.34 X_{3}-1.05 X_{1} X_{2}-0.48 X_{1} X_{3}-1.70 X_{2} X_{3}+1.95 X_{1}^{2}-2.74 X_{2}^{2}+3.02 X_{3}^{2}$ \\
$Y_{3}=95.57-1.72 X_{1}+10.42 X_{2}-0.65 X_{3}-2.10 X_{1} X_{2}-1.07 X_{1} X_{3}-0.73 X_{2} X_{3}+0.28 X_{1}^{2}-9.93 X_{2}^{2}+1.14 X_{3}^{2}$ \\
\hline
\end{tabular}

Table V. Standardized Main Effect of the Factors on the Responses

\begin{tabular}{|c|c|c|c|c|c|c|c|c|c|}
\hline & \multicolumn{3}{|c|}{ 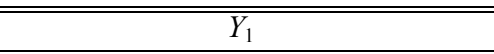 } & \multicolumn{3}{|c|}{$\overline{Y_{2}}$} & \multicolumn{3}{|c|}{$\overline{Y_{3}}$} \\
\hline & $\begin{array}{l}\text { Estimated } \\
\text { coefficient }\end{array}$ & P-value & $\begin{array}{l}\text { Standardized } \\
\text { main effect } \\
\text { (SME) }\end{array}$ & $\begin{array}{l}\text { Estimated } \\
\text { coefficient }\end{array}$ & P-value & $\begin{array}{l}\text { Standardized } \\
\text { main effect } \\
\text { (SME) }\end{array}$ & $\begin{array}{l}\text { Estimated } \\
\text { coefficient }\end{array}$ & P-value & $\begin{array}{c}\text { Standardized } \\
\text { main effect } \\
\text { (SME) }\end{array}$ \\
\hline$A_{1}$ & -5.39 & $<0.0001$ & -35.93 & -6.29 & $<0.0001$ & -19.66 & -1.72 & 0.0804 & -2.18 \\
\hline $\mathrm{A}_{2}$ & 0.41 & 0.0430 & 2.73 & 4.45 & $<0.0001$ & 13.91 & 10.42 & $<0.0001$ & 13.19 \\
\hline $\mathrm{A}_{3}$ & -2.11 & $<0.0001$ & -14.07 & -2.34 & 0.0007 & -7.31 & -0.65 & 0.4466 & -0.82 \\
\hline $\mathrm{A}_{4}$ & 0.36 & 0.1543 & 1.71 & -1.05 & 0.0646 & -2.33 & -2.10 & 0.1188 & -1.88 \\
\hline$A_{5}$ & 0.1 & 0.6586 & 0.48 & -0.48 & 0.3306 & -1.07 & -1.07 & 0.3804 & -0.96 \\
\hline$A_{6}$ & -0.86 & 0.0097 & -4.09 & -1.70 & 0.0124 & -3.78 & -0.73 & 0.5417 & -0.65 \\
\hline$A_{7}$ & 1.77 & 0.0005 & 8.05 & 1.95 & 0.0085 & 4.24 & 0.28 & 0.8190 & 0.24 \\
\hline$A_{8}$ & 0.49 & 0.0799 & 2.23 & -2.74 & 0.0020 & -5.96 & -9.93 & 0.0004 & -8.56 \\
\hline$A_{9}$ & 0.72 & 0.0229 & 3.27 & 3.02 & 0.0013 & 6.57 & 1.14 & 0.3703 & 0.98 \\
\hline
\end{tabular}

at the $5 \%$ significance level. The quadratic model generated by the design is of the form:

$$
\begin{aligned}
& \quad Y=A_{0}+A_{1} X_{1}+A_{2} X_{2}+A_{3} X_{3}+A_{4} X_{1} X_{2}+A_{5} X_{1} X_{3}+A_{6} X_{2} X_{3} \\
& +A_{7} X_{1}^{2}+A_{8} X_{2}^{2}+A_{9} X_{3}^{2}
\end{aligned}
$$

Where $A_{0}$ is an intercept and $A_{1}-A_{9}$ are the coefficients of

J. Pharm. Invest., Vol. 40, No. 5 (2010) respective factors and their interaction terms. Mathematical relationships in the form of quadratic equations for all responses and their standardized main effects (SME) are shown in Table IV and V, respectively. SME values were calculated by dividing the main effects by the standard error of the main effects. In addition, the contour plots and three- 
dimensional response surface plots were presented to estimate the effects of the independent variables on each response (Fig. 2).

As shown in Table $\mathrm{V}$, it can be noted that the statically significant coefficients ( $<<0.05)$ were $A_{1}, A_{2}, A_{3}, A_{6}, A_{7}$ and $A_{9}$ for $Y_{1} ; A_{1}, A_{2}, A_{3}, A_{6}, A_{7}, A_{8}$ and $A_{9}$ for $Y_{2}$; and $A_{2}$ and $A_{8}$ for $Y_{3}$, respectively. While the coefficients $A_{1}$ and $A_{3}$ demonstrated the antagonistic effects for both of $Y_{1}$ and $Y_{2}$, the coefficients $A_{2}$ showed the synergistic effect for both of $Y_{2}$ and $Y_{3}$. The largest SME of $A_{1}$ on the responses $Y_{1}$ and $Y_{2}$ indicated that the effect

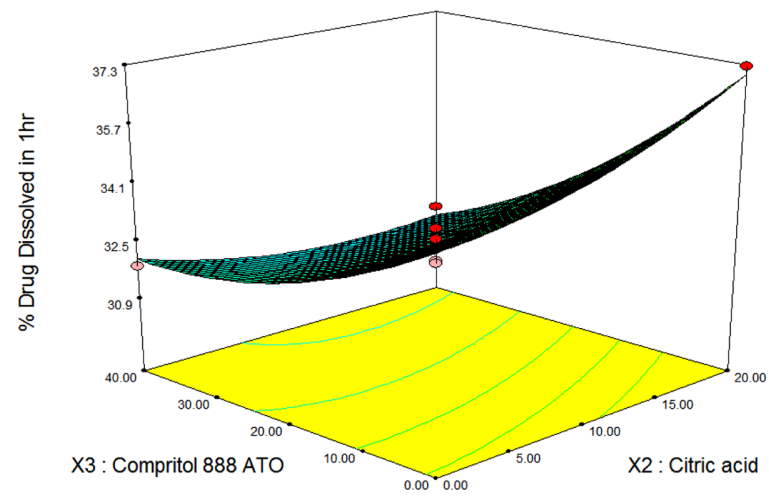

(a)

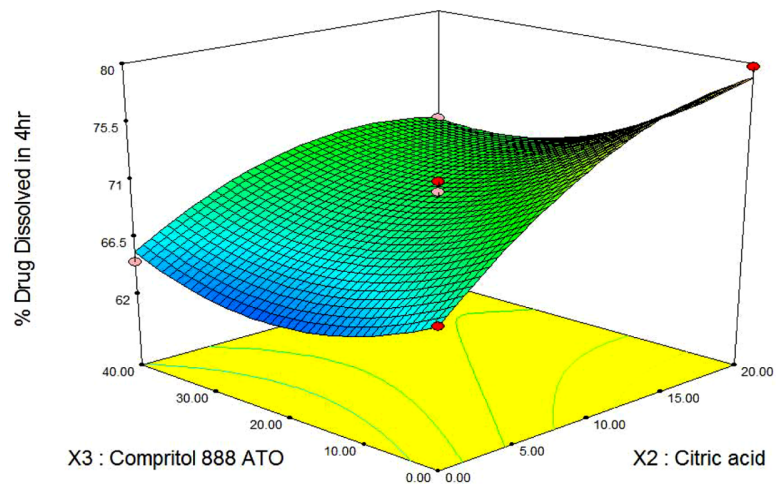

(b)

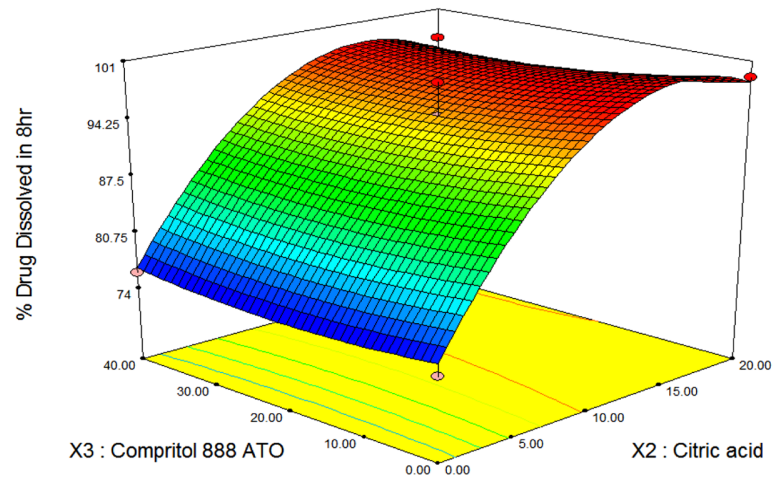

(c)

Figure 2. Response surface plots showing the cumulative \% drug dissolved after (a) $1 \mathrm{hr}$, (b) $4 \mathrm{hr}$ and (c) $8 \mathrm{hr}$. of PEO content $\left(X_{1}\right)$ was found to be the main influential factor in the drug release from matrix tablet. The decrease in drug release with increasing PEO content may be attributed to the increased in the viscosity of the gel layer as well as the formation of a gel layer with a thicker diffusional layer, as previously reported by many authors (Maggi et al., 2000; Maggi et al., 2002; Wu et al., 2005).

The drug release was also decreased with increasing content of Compritol 888 ATO $\left(X_{3}\right)$. The slower release from the matrices is due to almost complete coating of the drug particles by Compritol 888 ATO in the process of hot melt coating. In this case, it is expected that the penetration of the dissolution medium into the matrix will be low compared without Comrpitol 888 ATO.

In comparisons with the PEO $\left(X_{1}\right)$ and Compritol 888 ATO $\left(X_{3}\right)$ content, citric acid content $\left(X_{2}\right)$ showed significant synergistic effects on responses, $\mathrm{Y}_{2}$ and $\mathrm{Y}_{3}$. The addition of citric acid contributes to maintain a low $\mathrm{pH}$ inside the matrix and acts loosening the matrix structure through an increased porosity created after its dissolution and release (Rogelio et al., 2000). Therefore, drug release was increased with increasing content of citric acid $\left(X_{2}\right)$.

\section{Optimization}

In order to find the level of each independent variable that will lead to an optimized formulation, the optimization process was performed for $X_{1}, X_{2}$ and $X_{3}$ using the following target ranges; $30 \% \leq Y_{1} \leq 45 \% ; 70 \% \leq Y_{2} \leq 85 \% ; 85 \% \leq Y_{3} \leq 100 \%$. The target ranges of these responses were determined based on the dissolution profiles of the Wellbutrin ${ }^{\circledR}$ SR tablet, a commercial product. The optimization process was performed by graphical and numerical analysis using Design Expert software based on the methodology described by Myers and Montgomery (Myers and Montgomery, 2002). The optimized levels of PEO content $\left(X_{1}\right)$, citric acid content $\left(X_{2}\right)$ and Compritol 888 ATO $\left(X_{3}\right)$ were $12.5 \%, 2.5 \%$ and $10 \%$, respectively. Table VI shows the predicted and observed responses for the optimized formulations, indicating that the release profile of the bupro-

Table VI. Comparative Levels of Predicted and Observed Responses for Optimized Formulations

\begin{tabular}{cccc}
\hline \hline Response & $\begin{array}{c}\text { Predicted values } \\
(\%)\end{array}$ & $\begin{array}{c}\text { Observed vaules } \\
(\%)\end{array}$ & Predicted error $^{\mathrm{a}}$ \\
\hline$Y_{1}$ & 38.13 & 38.18 & 0.13 \\
$Y_{2}$ & 79.57 & 80.14 & 0.72 \\
$Y_{3}$ & 99.14 & 99.03 & -0.11 \\
\hline
\end{tabular}

aPredicted error $(\%)=($ observed value - predicted value $) /$ predicted value $\times 100 \%$ 


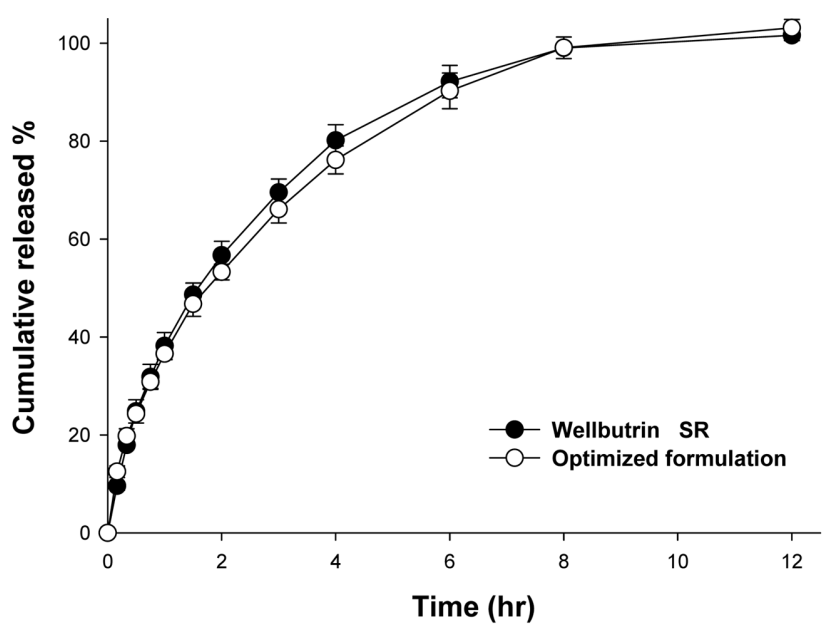

Figure 3. Dissolution profiles of optimized formulation in water $(\mathrm{n}=12$, means \pm S.D.).

pion hydrochloride matrix tablet with the optimized formulation was close to the predicted values. The dissolution profiles of the optimized formulation and commercial product are presented in Fig. 3. These dissolution profiles were compared using similarity factor $\left(f_{2}\right)$. The calculated value of $f_{2}$ was 79.83 , indicating that the dissolution profiles of the optimized formulation was comparable to those of the commercial Wellbutrin ${ }^{\circledR}$ SR tablet. In addition, the dissolution tests were performed at $\mathrm{pH} \mathrm{1.2,} 4.0$ and 6.8 buffers. When calculating the similarity factor $f_{2}$, values were 87.89, 72.56 and 69.37, respectively (Data not shown). All $f_{2}$ values were more than 50 ; this suggested that dissolution profiles of the matrix tablet were similar to those of Wellbutrin ${ }^{\circledR}$ SR in all four dissolution media.

\section{Conclusions}

The optimized formulation for bupropion hydrochloride was obtained with PEO, citric acid and Compritol 888 ATO using response surface methodology based on a Box-Behnken design. It was found that the optimized formulation was achieved with $12.5 \%$ PEO $\left(X_{1}\right), 2.5 \%$ citric acid $\left(X_{2}\right)$ and $10 \%$ Compritol 888 ATO $\left(X_{3}\right)$ and the observed responses were close to the predicted values for the optimized formulation. Furthermore, calculation of the similarity factors indicated that the dissolution of optimized formulation was similar to those of the commercial product Wellbutrin ${ }^{\circledR} \mathrm{SR}$.

\section{Acknowldgements}

This study was supported by a grant of the Korean Health Technology R\&D Project, Ministry for Health, Welfare \&
Family Affairs, Republic of Korea (A092018), and Priority Research Centers Program through the National Research Foundation of Korea (NRF) funded by the Ministry of Education, Science and Technology (2009-0093815).

\section{References}

Ascher, J.A., Cole, J.O., Colin, J., 1995. Bupropion : A review of its mechanism of antidepressant activity. J. Clin. Psychiat 56, 395-401.

Barrickman, L.L., Perry, P.J., Allen, A.J., Kuperman, S., Arndt, S.V., Herrmann, K.J., Schumacher, E., 1995. Bupropion versus methylphenidate in the treatment of attention deficit hyperactivity disorder. J. Am. Acad. Child Adolesc. Psychiatry 34, 649-657.

Cyr, M., Brown, C.S., 1998. Current drug therapy recommendations for the treatment of attention deficit hyperactivity disorder. Drugs 56, 215-223.

Ferris, R.M., Cooper, B.R., 1993. Mechanism of antidepressant activity of bupropion. J. Clin. Psychiatry Monograph 11, 2-14.

Ford, J.L., Rubinstein, M.H., McCaul, F., Hogan, J.E., Edgar, P.J., 1987. Importance of drug type, tablet shape and added diluents on drug release kinetics from hydroxypropylmethylcellulose matrix tablets. Int. J. Pharm. 40, 223-234.

Furlanetto, S., Cirri, M., Maestrelli, F., Corti, G., Mura, P., 2006. Study of formulation variables influencing the drug release rate from matrix tablets by experimental design. Eur. J. Pharm. Biopharm. 62, 77-84.

González, I.M., Robles, L.V., 2003. Influence of enteric citric acid on the release profile of 4-aminopyridine from HPMC matrix tablets. Int. J. Pharm. 251, 183-193.

Jamzad, S., Fassihi, R., 2006. Development of a controlled release low dose class II drug-Glipizide. Int. J. Pharm. 312, 24-32.

Jefferson, J.W., Pradko, J.F., Muir, K.T., 2005. Bupropion for major depressive disorder: Pharmacokinetic and formulation considerations. Clin. Ther. 27, 1685-1695.

Kranz, H., Brun, V.L., Wagner, T., 2005. Development of a multi particulate extended release formulation for ZK 811 752, a weakly basic drug. Int. J. Pharm. 299, 84-91.

Maggi, L., Bruni, R., Conte, U., 2000. High molecular weight polyethylene oxides (PEOs) as an alternative to HPMC in controlled release dosage forms. Int. J. Pharm. 195, 229-238.

Maggi, L., Segale, L., Torre, M.L., Ochoa M.E., Conte, U., 2002. Dissolution behaviour of hydrophilic matrix tablets containing two different polyethylene oxides (PEOs) for the controlled release of a water-soluble drug. Dimensionality study. Biomaterials 23, 1113-1119.

Moore, J.W., Flanner, H.H., 1996. Mathematical comparison of dissolution profiles. Pharm. Tech. 20, 64-74.

Myers, R.H., Montgomery, D.C., 2002. Response Surface Methodology: Process and Product Optimization Using Designed Experiments. John Wiley \& Sons, New York, pp. 273-286. Palamara, K.L., Mogul, H.R., Peterson, S.J., Frishman, W.H., 
2006. Obesity: new perspectives and pharmacotherapies. Cardiol. Rev. 14, 238-258.

Settle, E.C., 1998. Bupropion sustained release : side effect profile. J. Clin. Psychiatry 59, 32-36.

Wu, N., Wang, L.S., Tan, D.C., Moochhala, S.M., Yang, Y.Y.,
2005. Mathematical modeling and in vitro study of controlled drug release via a highly swellable and dissoluble polymer matrix: polyethylene oxide with high molecular weights. J. Control. Release 102, 569-581. 